\title{
The Fabrication and Characterization of PCL/Rice Husk Derived Bioactive Glass-Ceramic Composite Scaffolds
}

\author{
Farnaz Naghizadeh, ${ }^{1}$ Naznin Sultana, ${ }^{1}$ Mohammed Rafiq Abdul Kadir, ${ }^{1}$ \\ Tengku Muzaffar Tengku Md Shihabudin, ${ }^{2}$ Rafaqat Hussain, ${ }^{3}$ and Tunku Kamarul ${ }^{4}$ \\ ${ }^{1}$ Department of Clinical Sciences, Faculty of Biosciences and Medical Engineering, Universiti Teknologi Malaysia, 81310 Johor, Malaysia \\ ${ }^{2}$ Department of Orthopaedics, School of Medical Sciences, Universiti Sains Malaysia, Kubang Kerian Campus, \\ 16150 Kubang Kerian, Kelantan, Malaysia \\ ${ }^{3}$ Department of Chemistry, Faculty of Science, Universiti Teknologi Malaysia, 81310 Johor, Malaysia \\ ${ }^{4}$ Tissue Engineering Group, NOCERAL, Department of Orthopaedic Surgery, University of Malaya, 50603 Kuala Lumpur, Malaysia
}

Correspondence should be addressed to Mohammed Rafiq Abdul Kadir; rafiq@biomedical.utm.my

Received 2 September 2013; Revised 10 November 2013; Accepted 5 December 2013; Published 16 January 2014

Academic Editor: Shafiul Chowdhury

Copyright (C) 2014 Farnaz Naghizadeh et al. This is an open access article distributed under the Creative Commons Attribution License, which permits unrestricted use, distribution, and reproduction in any medium, provided the original work is properly cited.

\begin{abstract}
The present study was conducted to fabricate a 3D scaffold using polycaprolactone (PCL) and silicate based bioactive glass-ceramic (R-SBgC). Different concentrations of R-SBgC prepared from rice husk ash (RHA) were combined with PCL to fabricate a composite scaffold using thermally induced phase separation (TIPS) method. The products were then characterized using SEM and EDX. The results demonstrated that R-SBgC in PCL matrix produced a bioactive material which has highly porous structure with interconnected porosities. There appears to be a relationship between the increase in $\mathrm{R}-\mathrm{SBgC}$ concentration and increased material density and compressive modulus; however, increasing R-SBgC concentration result in reduced scaffold porosity. In conclusion, it is possible to fabricate a PCL/bioactive glass-ceramic composite from processed rice husk. Varying the R-SBgC concentrations can control the properties of this material, which is useful in the development of the ideal scaffold intended for use as a bone substitute in nonload bearing sites.
\end{abstract}

\section{Introduction}

The use of biomaterials for treating bone defects has been widely accepted despite the availability of natural bone grafts. This is due to the lack of donor site morbidity issues and the avoidance of possible disease transmission regarding biomaterials [1-3]. Among the many biomaterials available, polycaprolactone (PCL), which is a synthetic biodegradable polymer, has shown great promise $[4,5]$. In fact, PCL is presently one of the few biomaterials approved by FDA for use as bone graft substitutes [5-7]. PCL can be easily fabricated into a material possessing the desired toughness, which is appropriate for bone replacement. In addition to being biocompatible, PCL has a low degradation rate, which is a desired feature in a biomaterial designed for specific sites of bone with longer healing times [4]. However, this material alone without additives demonstrates low mechanical resistance to compressive loading, hydrophobicity, and low bioactivity [8]. To counter these problems, bioactive ceramics/biodegradable polymer composite materials were introduced. These new materials demonstrated superior properties including improvement in material strength, stiffness, biodegradability, osteoconductivity, and bioactivity [4, $6,7,9]$. In addition, polymer/bioactive ceramic composite scaffolds have structures that resemble bone, where the inorganic component of these scaffolds mimics the hydroxycarbonate-apatite (HCA) motifs while the polymer component mimics the collagen-rich extracellular matrix. Although both HCA and bioactive glasses have been used to form composites with superior bioactivity, bioactive glasses have been preferred due to their superior osteoconductive properties $[10,11]$. Furthermore, not only do bioactive glasses 
increase the hydrophilicity but they also increase the hydrolysis of PCL, thereby increasing the composites degradation rate, albeit at a control rate sufficient for creeping substitution to occur $[4,5,12-15]$. Along with other Added features such as improved porosity with interconnectivity of the pores in this composite, such a product will be of great value for use in clinical applications. A method by which increased porosity can be achieved is adopting thermally induced phase separation technique (TIPS) [16]. Despite its many advantages, the fabrication of such composites has not been widely described in the literature. We therefore conducted a study to determine if $\mathrm{R}-\mathrm{SBgC}$ made from rice husk, which is an abundant plant waste produced in most parts of the world, can be fabricated to become a composite PCL scaffold with the desired properties required for use as a potential bone substitute.

\section{Materials and Methods}

2.1. Materials Preparation. PCL (molecular weight of 70,00090,000) was purchased from Sigma Aldrich. Analytical grade 1, 4-Dioxane was used as a solvent (Sigma Aldrich.). The RSBgC was synthesized by sol-gel method using RHA with the composition of (mol\%) $50 \mathrm{SiO}_{2} \cdot 25 \mathrm{Na}_{2} \mathrm{O} \cdot 25 \mathrm{CaO}$ [17]. Rice husk was obtained from Kahang Organic Rice Eco Farm in Johor and after washing and drying in a hot air oven overnight, it was heat treated in $650^{\circ} \mathrm{C}$ to achieve RHA with $98 \%$ pure Silica $[17,18]$. Sol preparation involved mixing adequate amounts of distilled water, $\mathrm{NaOH}$ pellets (Merck.), and RHA. This was done under stirring condition, until a clear solution was obtained. This Process generally takes about one hour. Acidic solutions of $\mathrm{HNO}_{3}\left(2 \mathrm{~N}\right.$ ) with $\mathrm{CaNO}_{3} \cdot 4 \mathrm{H}_{2} \mathrm{O}$ (all Merck.) were mixed separately. The prepared sodium silicate solution was slowly added to the acidic solution until the gelling process was completed. The gel was allowed to age and dry, and later it was left in the furnace to sinter for 1 hour at $600^{\circ} \mathrm{C}$. Lastly, the product was crushed to obtain the $\mathrm{R}-\mathrm{SBgC}$ in the powder form. The amorphous and partially crystallized phases of R-SBgC were confirmed using X-ray diffraction (XRD) through $\mathrm{CuK} \alpha$ radiation (Rigaku Geigerflex Dmax-C). To achieve homogenous nanosized particles in the powder, the dried and sintered R-SBgC was freeze-dried using the proper related apparatus (LABCONCO-Freeze dry system, USA).

2.2. Scaffold Fabrication. The TIPS technique was utilized to fabricate R-SBgC/PCL composite scaffold as shown in Figure 1. $15 \mathrm{~mL}$ of 1, 4 Dioxane was used to dissolve $1 \mathrm{~g}$ of PCL polymer granules (PCL concentration \%6.6 (w/v)). The R-SBgC was dispersed in the polymer solution in $10 \%$, $20 \%$, and $30 \%(\mathrm{w} / \mathrm{w})$ and homogenized using a hand-held homogenizer. The polymer/R-SBgC solutions as well as the pure PCL solution were poured in separate glass vials and stored in a $-18^{\circ} \mathrm{C}$ freezer. After overnight freezing, the materials were placed in a freeze-dryer for 48 hours subjected to $-45^{\circ} \mathrm{C}$ and 0.012 mbar pressure in order to eliminate the solvent. The obtained materials were then left at room temperature for 24 hours.

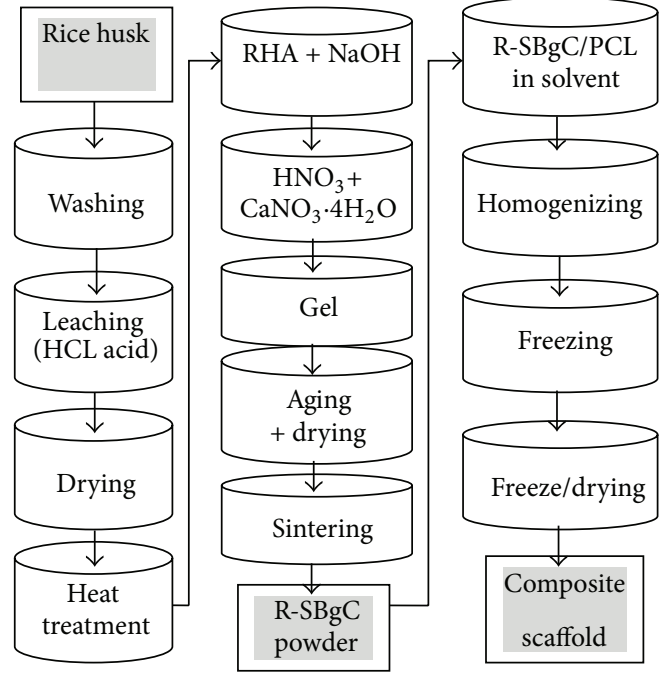

FIGURE 1: Schematic diagram of the scaffold production.

\subsubsection{Characterization Using SEM and FESEM and EDX} before and after Bioactivity Testing. To study the morphology of the composite scaffolds, scanning electron microscopy (SEM; Hitachi, TM 3000) and field emission scanning electron microscopy (FESEM; Carls Zeiss 35 VP Supra, Germany) were employed with acceleration voltages of $15 \mathrm{kV}$ and $20 \mathrm{kV}$, respectively. After freezing for 24 hours at $-18^{\circ} \mathrm{C}$, the materials were cut into desired shapes. Specimens were sputter-coated with a thin layer of gold prior to SEM. Energy dispersive X-ray spectroscopy (EDX; Carls Zeiss 35 VP Supra, Germany) was utilized to ascertain the presence of R-SBgC nanoparticles distribution in PCL matrix and hydroxy apatite (HA) formation. Testing was done before and after bioactivity testing to determine structural changes as a result of the simulated bioactivity in vitro.

2.2.2. Determination of Density and Porosity. The density and porosity of the polymeric foams were measured using liquid displacement method [19]. Usually, water is used as the displacement liquid. However, in the present study, ethanol was instead utilized because (1) ethanol, like water, does not dissolve PCL and (2) ethanol has the ability to infiltrate into the pores more easily without causing any shrinkage or swelling effects on the scaffolds.

Measurement for density and porosity of the scaffolds are described as follows. The Scaffold sample (weight $=W$ ) was immersed in a graduated cylinder containing a known volume of ethanol $\left(V_{1}\right)$ for $15 \mathrm{~min}$. The total volume of ethanol and the ethanol-impregnated scaffold was referred to as $V_{2}$ and the remaining volume of ethanol after removing impregnated scaffold was labeled $V_{3}$. The scaffold volume was obtained by subtracting $V_{2}$ from $V_{1}$. The volume difference between $V_{1}$ and $V_{3}$ indicates the volume of ethanol held inside the foams. The total volume of the foam is expressed as

$$
V=\left(V_{2}-V_{1}\right)+\left(V_{1}-V_{3}\right)=V_{2}-V_{3} \text {. }
$$



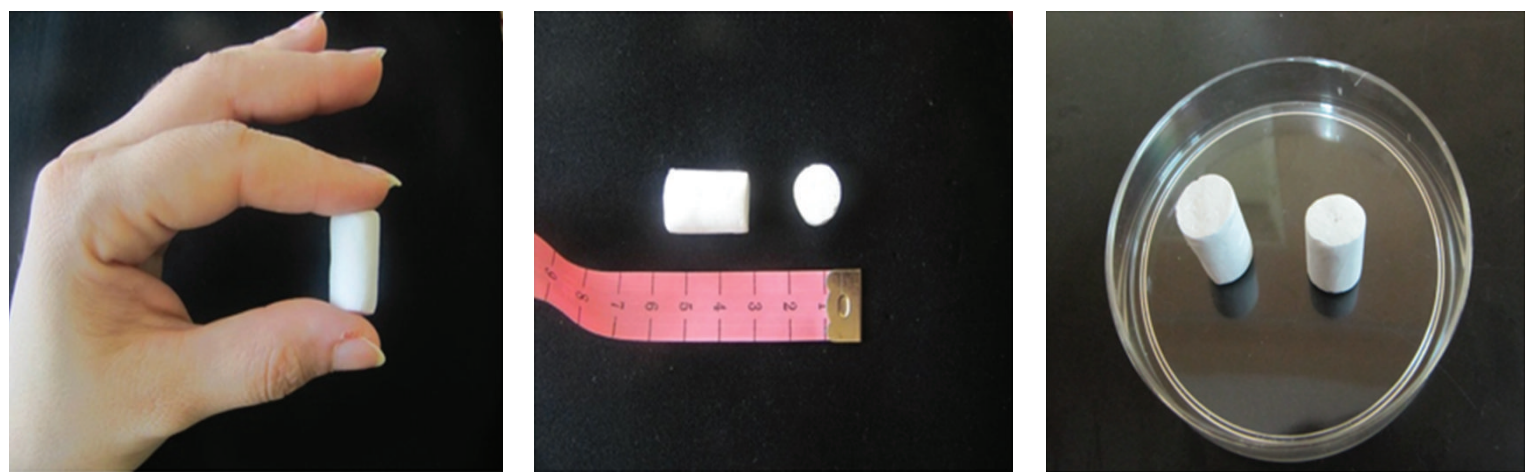

Figure 2: Physical appearance of R-SBgC/PCL scaffolds.

Whilst the density of the foam is

$$
d=\frac{W}{\left(V_{2}-V_{3}\right)},
$$

and porosity is

$$
P=\frac{\left(V_{1}-V_{3}\right)}{\left(V_{2}-V_{3}\right)} .
$$

Equations (1), (2), and (3) were used for calculating the density and porosity. The calculated results are presented as a mean \pm standard deviation (SD) for each group of scaffolds. To determine the pore size, the average of measurements using SEM images of 25 random pores from each scaffold was calculated.

2.2.3. Capillarity Test. The scaffold permeability was determined by performing capillarity test. The fluid infiltrated the interconnected pores due to the capillarity effect. For this assessment, a lean slice of composite scaffold ( $1 \mathrm{~mm}$ thick) was placed into a solution with a viscosity similar to that of human blood. The solution colored by adding some drops of bluish purple ink helps track the fluid movement.

2.2.4. Compression Mechanical Test. To evaluate the compressive mechanical properties of the composite scaffolds, a universal testing machine (Instron 5982, USA) at a crosshead velocity of $0.6 \mathrm{~mm} / \mathrm{min}$ with a $1000 \mathrm{~N}$ load cell was used. At least 5 specimens of each scaffold were prepared in cylindrical shape each with a $10 \mathrm{~mm}$ diameter and $5 \mathrm{~mm}$ height. From the initial linear region of the plotted stress-strain curve, the compressive modulus was calculated for each scaffold. For each group of scaffolds, five samples were evaluated and the results are presented as a mean \pm SD.

2.2.5. In Vitro Bioactivity Testing. In vitro bioactivity assessment were accomplished using standard SBF following the method described by Kokubo and Takadama [20]. The SBF solution was prepared through dissolving particular amounts of $\mathrm{NaCl}, \mathrm{KCl}, \mathrm{CaCl}_{2}, \mathrm{NaHCO}_{3}, \mathrm{~K}_{2} \mathrm{HPO}_{4} \cdot 3 \mathrm{H}_{2} \mathrm{O}$, $\mathrm{MgCl}_{2} \cdot 6 \mathrm{H}_{2} \mathrm{O}$, and $\mathrm{Na}_{2} \mathrm{SO}_{4}$ into deionized water. The solution was adjusted to reach a $\mathrm{pH}$ 7.3-7.4 by altering the amount of Tris buffer and 1.0 M-HCl [20]. Scaffolds were cut to thin slices with a diameter of $10 \mathrm{~mm}$ and height of $3 \mathrm{~mm}$. These scaffolds were then immersed in $50 \mathrm{~mL}$ of prepared standard $\mathrm{SBF}$ in centrifuge tubes at $37^{\circ} \mathrm{C}$ incubated water bath for 14 and 21 days. The SBF solution was refreshed once a week until completion. The PCL/R-SBgC composite in contact with SBF was then analyzed using a FESEM and an EDX to determine the presence of Ca-P phase's formation on the surface of the composite scaffold.

\section{Result and Discussion}

3.1. R-SBgC Powder. Figure 3 illustrates the FESEM image and EDX spectrum of the synthesized R-SBgC powder prior to being added into the scaffold mixture. The FESEM analysis of the R-SBgC powder confirmed the formation of spherical agglomerated particles with diameter ranging from 64 to $300 \mathrm{~nm}$ with a mean diameter of $120 \mathrm{~nm}$ via randomly measuring particle size at 25 different locations. The EDX spectrum (Figure 3(b)) confirmed the presence of $\mathrm{Si}, \mathrm{Ca}$, and $\mathrm{Na}$ as the main elements of the R-SBgC composition.

3.2. Fabrication of the 3D Porous Composite Scaffold. This study demonstrated that a $3 \mathrm{D}$ highly porous structure of PCL/R-SBgC composite scaffold can be produced using TIPS technique. It is said that porosity can be controlled by altering polymer content, polymer concentration, and solvent concentration as well as other additive phase parameters [21]. In this study different concentrations of PCL including 5, 6.6 and $10 \%(\mathrm{w} / \mathrm{v})$ were tested; the optimized concentration of PCL $6.6 \%(\mathrm{w} / \mathrm{v})$ was chosen because it produced the best porosity up to $80 \%$ in the material. Based on previous reports, it is believed that homogenous distribution of bioactive ceramics (bioactive glass) in polymer solution and in scaffold has direct effects on the bioactivity and mechanical properties of scaffolds $[12,22]$. This may be due to the higher concentration of doped bioactive glass, which tends to obstruct the pores $[12,13]$. In this study, R-SBgC in $10 \%, 20 \%$, and maximum $30 \%(\mathrm{w} / \mathrm{w})$ were incorporated to the scaffold and homogenized to achieve fine distribution in polymer matrix. According to the literature, the use of R-SBgC nanoparticles is supposed to be useful for further in vitro tests because of its high surface area to volume ratio, which is said to enhance 


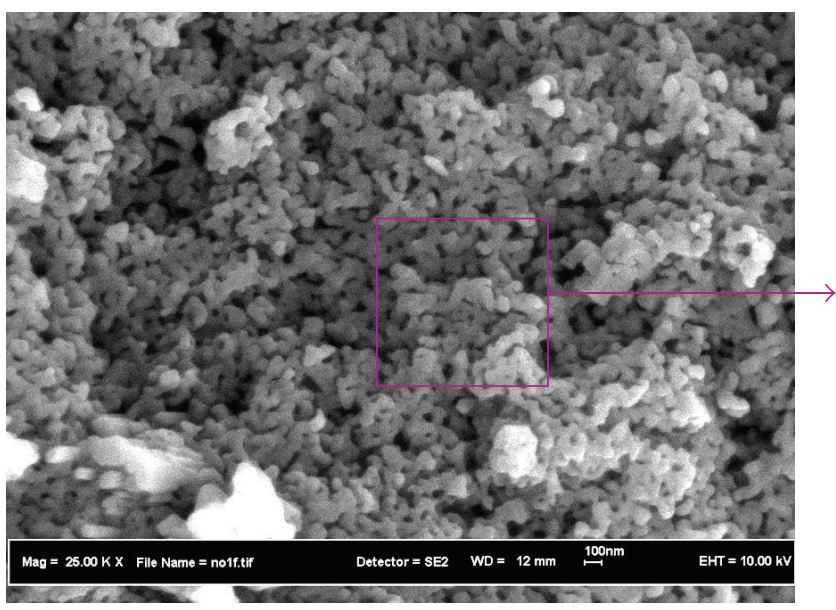

(a)

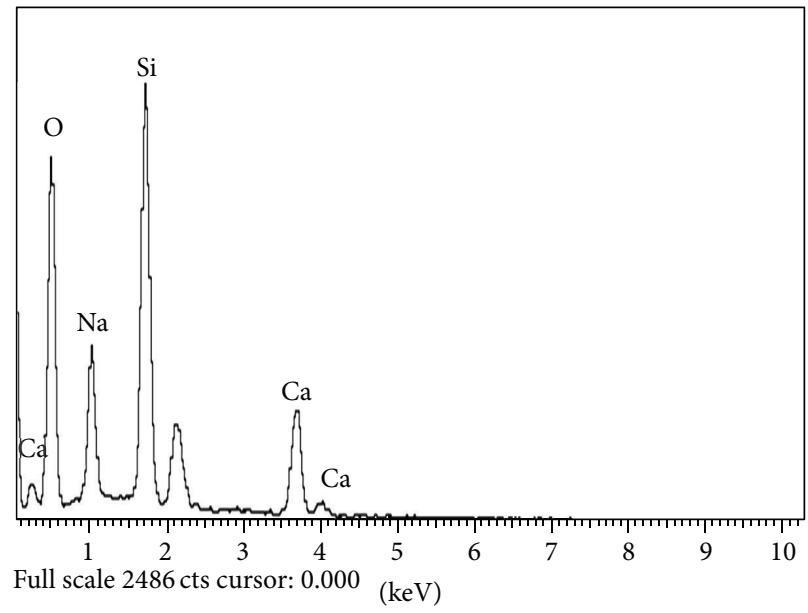

(b)

FIGURE 3: FESEM micrographs of R-SBgC in magnification 25K X; marker $100 \mathrm{~nm}$ (a) and an EDAX spectrum of R-SBgC nanopowder (b).

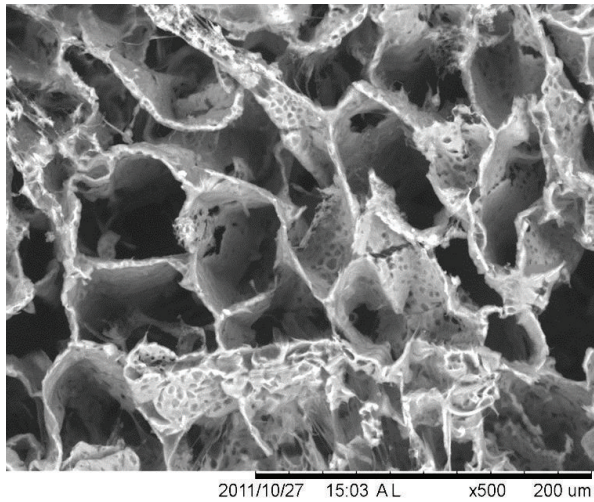

(a)

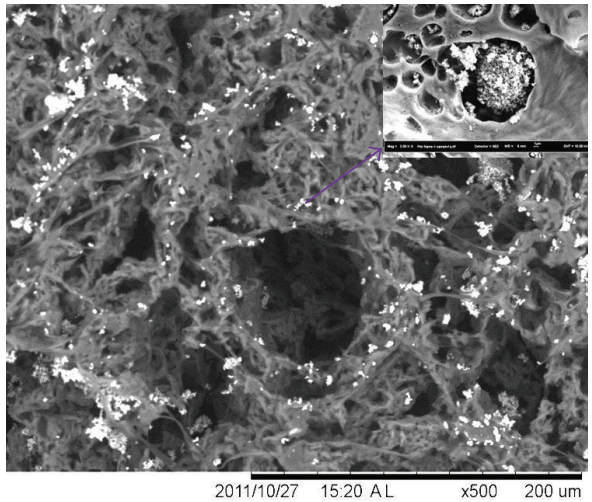

(b)

FIGURE 4: SEM micrographs of the porous structure of scaffolds were made using the $6.6 \%$ (w/v) PCL polymer solution. (a) Pure PCL scaffold, (b) $30 \% \mathrm{R}-\mathrm{SBgC} / \mathrm{PCL}$ scaffold in magnification 500x; marker $200 \mu \mathrm{m}$. A higher magnification image (2K X) in upper right section of (b) illustrates an aggregate trapped in the porosity including nanoparticles of R-SBgC powder.

cell adhesion [23]. The physical appearance of the composite scaffold fabricated by TIPS technique can be seen in Figure 2. This figure illustrates the homogeneity and proper size of the fabricated scaffolds, which could be handled and carried without difficulty.

\subsection{Characterization}

3.3.1. SEM Micrographs, FESEM, and EDX Analysis prior to Bioactivity Testing. SEM micrographs reveal porous structures within the surfaces and inner structures of the fabricated scaffolds. SEM images of pure PCL scaffold and $30 \%(\mathrm{w} / \mathrm{w}) \mathrm{R}-\mathrm{SBgC} / \mathrm{PCL}$ composites are presented in Figure 4 as representatives of minimum and maximum $\mathrm{R}-\mathrm{SBgC}$ content scaffolds. The morphologies of the pure PCL scaffold and composite present a typical microstructure of Polymeric scaffold prepared using TIPS technique [23]. Furthermore, the fabricated composite scaffold in this study showed an average pore size of $100 \mu \mathrm{m}$ with interconnected porous structure which is deemed suitable for cell migration and penetration [24]. In Figures 4(a) and 4(b), the pore size appears to be reduced with increasing amount of $\mathrm{R}-\mathrm{SBgC}$. El-Kady et al. also report that the increase in the glass content from $25 \mathrm{wt} \%$ to $50 \mathrm{wt} \%$ resulted in a decrease of composite scaffold pore size [13]. This appears to be supported by other studies [13, 25]. Another explanation for the reduction in pore size may be the decrease in solvent volume relative to the total weight of the polymer and bioactive glass nanoparticles during the preparation of the composites [12]. In addition to reduced pore sizes, there appears to be increased agglomeration cluster formation with increasing R-SBgC concentration. This is especially evident in composites of $30 \% \mathrm{R}-\mathrm{SBgC}$ doped scaffold (Figure 4(b)). Although most of the R-SBgC nanoparticles are distributed homogeneously on the pore walls of the scaffolds, some aggregates of nanoparticles are detectible in some pores of the scaffold as observed in higher magnification $(2 \mathrm{~K} \mathrm{X})$ in Figure 4(b). The presence of R-SBgC particles was confirmed by the EDX data as presented in Figure 5. 
TABLE 1: Density porosity, pore size, and compressive modulus of R-SBgC/PCL composite scaffolds.

\begin{tabular}{lcccc}
\hline Scaffold \% (w/w) & Density $^{*}\left(\mathrm{~g} / \mathrm{cm}^{3}\right)$ & Porosity $^{*}(\%)$ & Average pore sizes $(\mu \mathrm{m})$ & Compressive modules $^{*}(\mathrm{MPa})$ \\
\hline $0 \%$ R-SBgC/PCL & $0.062 \pm 0.01$ & $80 \pm 0.03$ & 105 & $0.375 \pm 0.02$ \\
$10 \%$ R-SBgC/PCL & $0.078 \pm 0.05$ & $78 \pm 0.02$ & 102 & $0.411 \pm 0.06$ \\
$20 \%$ R-SBgC/PCL & $0.101 \pm 0.01$ & $77 \pm 0.03$ & 100 & $0.442 \pm 0.07$ \\
$30 \%$ R-SBgC/PCL & $0.163 \pm 0.06$ & $76 \pm 0.04$ & 98 & $0.475 \pm 0.04$ \\
\hline
\end{tabular}

${ }^{*}$ Mean \pm standard deviation; $n=5$.

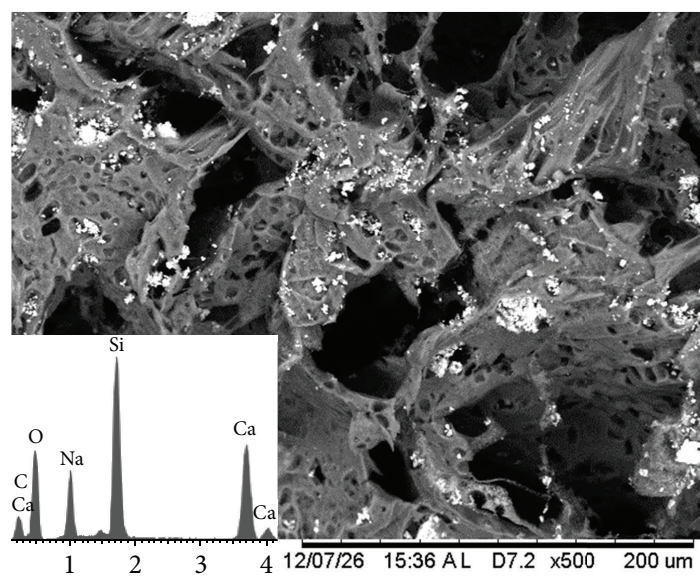

Figure 5: An EDX spectrum confirming the presence of R-SBgC particles in 20\% R-SBgC/PCL composite scaffold.

3.3.2. Scaffold's Density and Porosity. The porosity and density results of this study are summarized in Table 1 . These findings appear to be in line with that of the SEM observation. It was found that by increasing $\mathrm{R}-\mathrm{SBgC}$ concentration, the porosity in the scaffold is maintained but with reduced pore sizes. The degree of porosity percentage decreased with the increase in $\mathrm{R}-\mathrm{SBgC}$ concentration from $10 \%$ to $30 \%(\mathrm{w} / \mathrm{w})$. Porosity of materials varies from $80 \%$ to $76 \%$ for pure polymer and 30\% (w/w) R-SBgC/PCL scaffolds respectively. Findings from studies such as Hong et al. appears to support our findings [12]. The average pore sizes in the tested materials ranged between 98 and $105 \mu \mathrm{m}$ depending on the $\mathrm{R}-\mathrm{SBgC}$ concentration. These sizes appear to be what is recommended for bone tissue engineering purposes [21, 24].

3.3.3. Compression Test. The enhancement of the mechanical performance due to the introduction of inorganic nanoparticles in composites has been intensively discussed in previous studies [12, 21, 26]. Hong et al. [12] reported that mechanical properties of PLLA matrix can be improved by adding bioglass ceramic (BGC) nanoparticles. Similarly, the improvement in the elastic modulus in the present material was supported by data presented elsewhere [23]. It has been previously suggested that the addition of HA or bioactive glasses nanoparticles enhances the fortification of the scaffold material which is seen as the increase in structural strength $[21,26]$. Increasing the R-SBgC concentration increases the compressive modulus of the composite scaffolds (Table 1). Scaffold with $30 \% \mathrm{R}-\mathrm{SBgC} / \mathrm{PCL}$ showed a higher resistance to compressive deformation compared to scaffold made of pure PCL (Figure 7). The initial slope of the stress-strain curve was used to obtain the compressive modulus of scaffolds. This method was similar to that used in other studies [21]. Totally, $26.6 \%$ increase in the compressive modulus was observed when $30 \% \mathrm{R}-\mathrm{SBgC}$ was incorporated into the pure PCL scaffold (Table 1). The compressive modulus increased from $0.375 \mathrm{Mpa}$ to $0.475 \mathrm{MPa}$ when the R-SBgC concentration increased from $0 \%$ to $30 \%(\mathrm{w} / \mathrm{w})$, respectively. This was an increase compared to a previous published report which demonstrated a compressive strength increase from 0.28 to $0.35 \mathrm{MPa}$ as the BGC content increased from 0 to $30 \mathrm{wt} \%$. [12].

3.3.4. Capillarity Test. To investigate the pores interconnection of composite scaffold, capillarity test was carried out on $30 \% \mathrm{R}-\mathrm{SBgC} / \mathrm{PCL}$ composite scaffold which is supposed to have smaller pore size and some more blocking site by $\mathrm{R}-\mathrm{SBgC}$ particles. The sequence of events can be seen in photographs presented in Figure 8 showing clear infiltration of the colored liquid through the porous network of the composite scaffold. The colored fluid appeared to escalate upwards in the scaffold within seconds, thus indicating the porosity with possible interconnectivity within this material. This finding was similar to those demonstrated in other porous scaffold structures reported previously [27].

3.3.5. In Vitro Bioactivity Evaluation. The bioactivity evaluation of composite scaffolds was performed via SBF test. This involved the immersion of the tested materials in SBF for up to 21 days. The evaluation of bioactivity involves the use of SEM and EDX as shown in Figures 6(a)-6(h). The SEM micrographs revealed that all R-SBgC that included scaffolds induced the microsized apatite formation after SBF immersion. This finding was supported by the EDX data. Pure PCL scaffold illustrated no major change in 14 and 21 days; however, by 14 days, a small amount of $\mathrm{NaCl}$ precipitation on the surfaces of scaffold could be observed and proved by EDX data (Figure 6(a)). Analysis of samples after 21 days immersion showed low intensity peaks of $\mathrm{CaCl}_{2}$ and $\mathrm{NaCl}$, which can be recognized by the tiny distributed light spots under SEM (Figure 6(b)). This finding was proved by EDX data as well. The unknown peak of EDX micrographs is related to $\mathrm{Au}$ coating for sample preparation before using SEM. Results for $10 \%$ (w/w) R-SBgC/PCL scaffold after 14 days revealed precipitation of $\mathrm{Ca}, \mathrm{P}, \mathrm{Na}, \mathrm{Cl}$, and $\mathrm{Si}$ as shown in Figure 6(c). EDX results confirmed the presence of $\mathrm{Si}$ as one of the main components of $\mathrm{R}-\mathrm{SBgC}$ powder, 


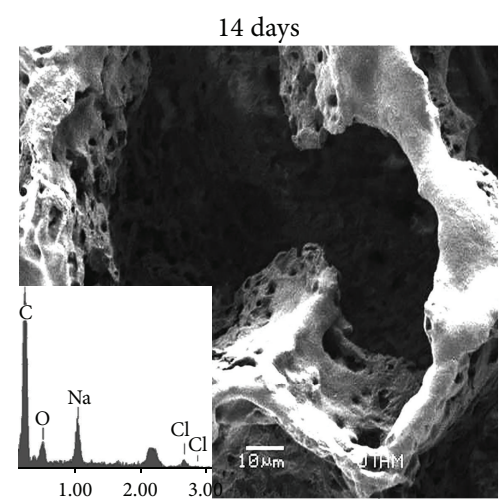

(a)

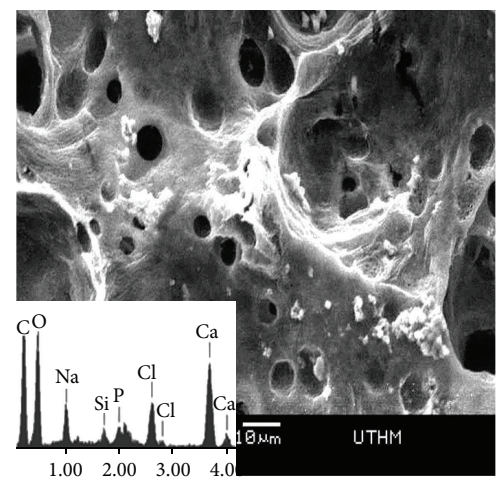

(c)

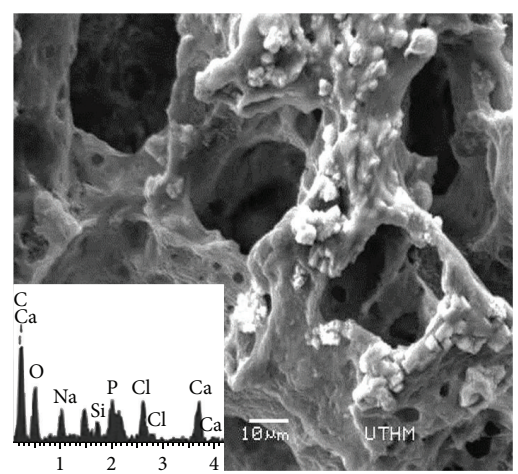

(e)

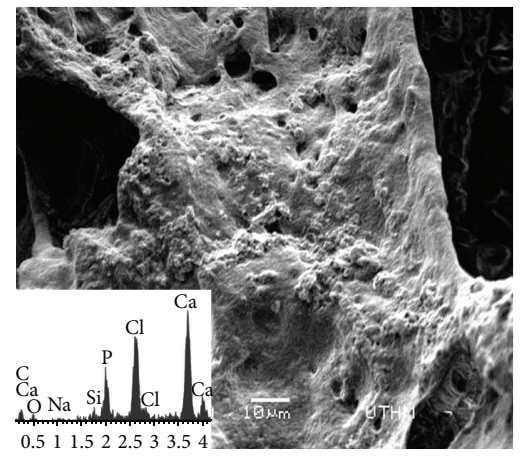

(g)

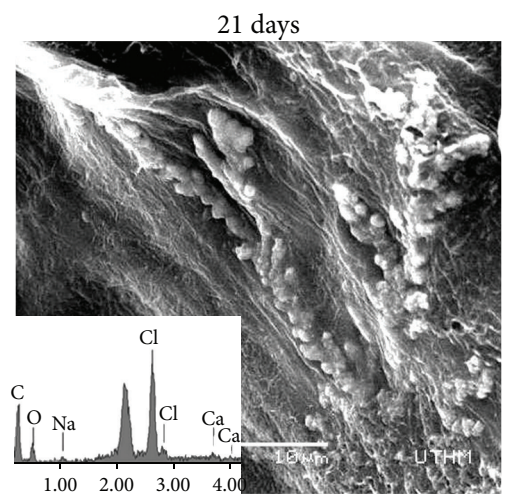

(b)

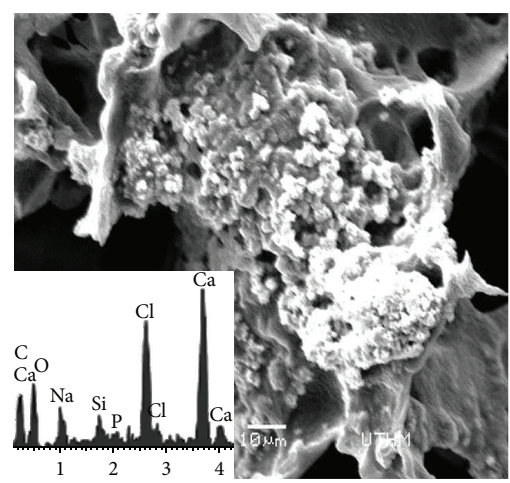

(d)

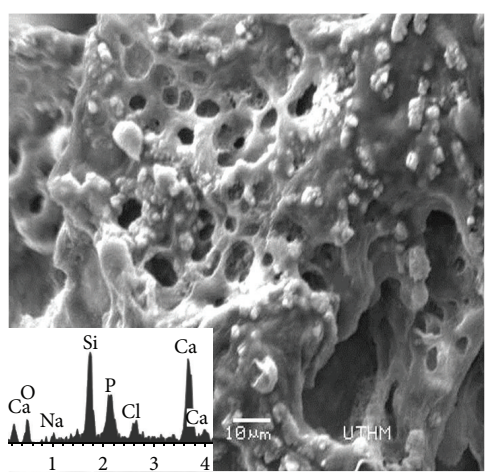

(f)

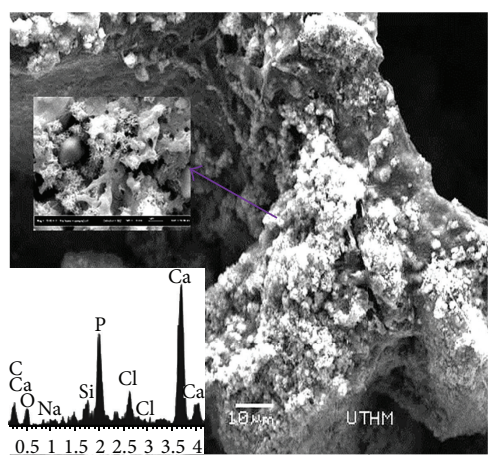

(h)

FIGURE 6: SEM micrographs and EDX spectra illustrating the change on the surface of the scaffolds after 14 and 21 days (left and right columns illustrate the images for 14 and 21 days, resp.) immersion in SBF (a-b) pure PCL scaffold (c-d) 10\% (w/w) R-SBgC/PCL composite scaffold and (e-f) 20\% R-SBgC/PCL scaffold and (g-h) 30\% R-SBgC/PCL scaffold in magnification 1000x; marker $10 \mu \mathrm{m}$. The image in upper left of section (h) illustrates the small HA crystals with needles like structure on the surface of scaffold in higher magnification (10K X). 


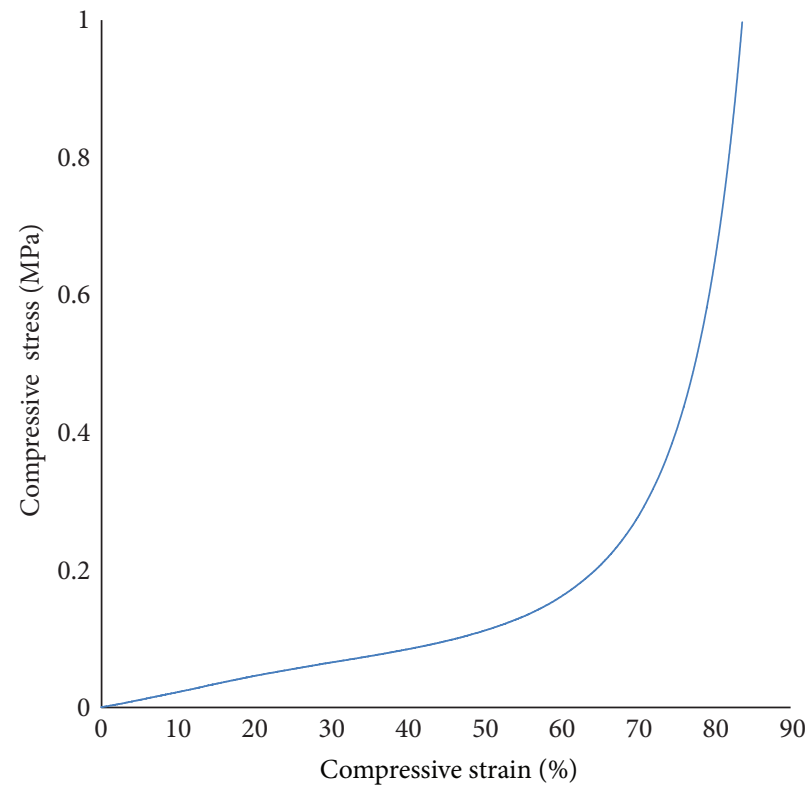

(a)

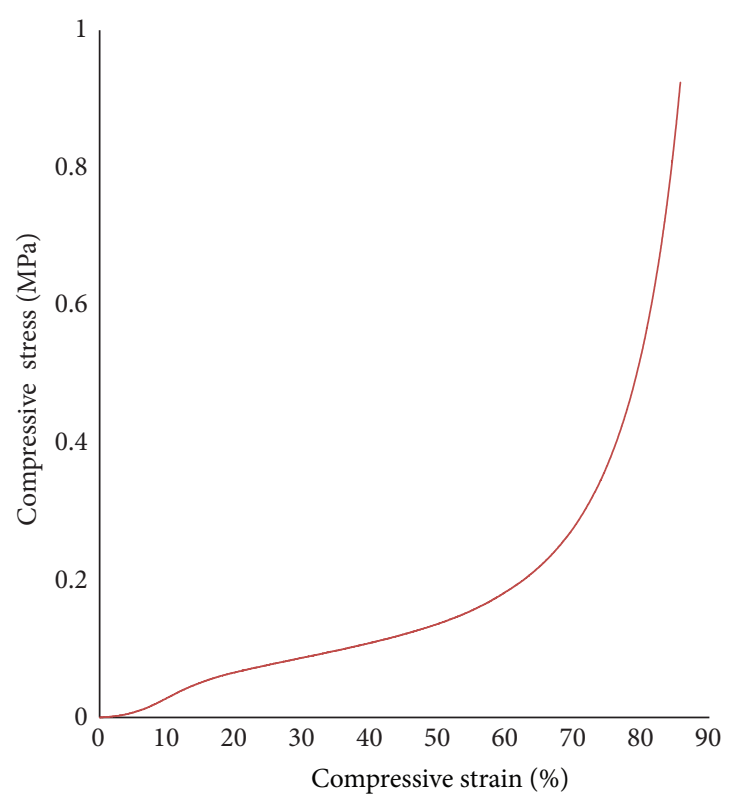

(b)

Figure 7: Compressive properties of PCL and R-SBgC/PCL scaffolds. (a) Typical compressive stress/strain curves of PCL, (b) compressive module of $30 \%(w / w)$ R-SBgC/PCL composite scaffold.

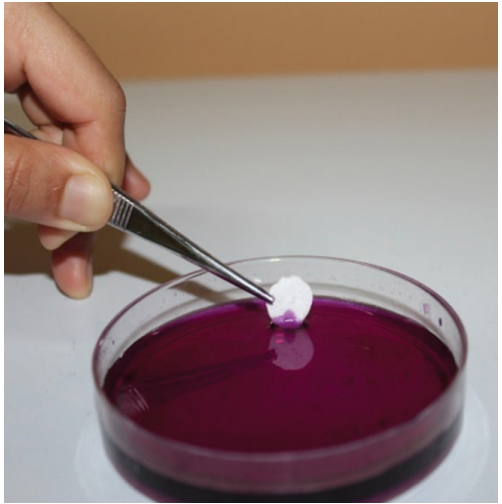

(a)

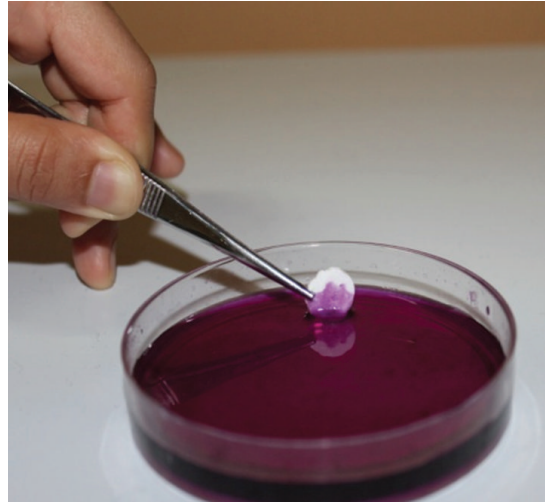

(b)

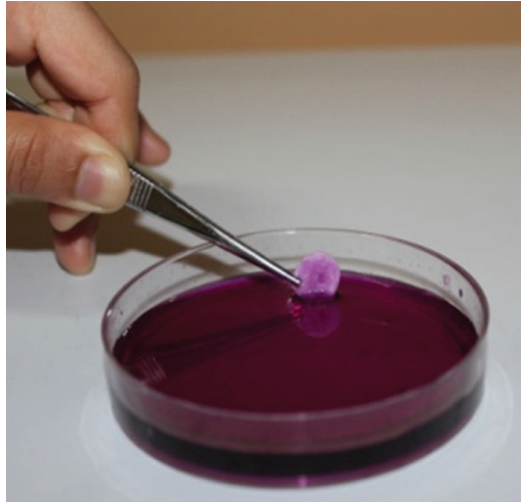

(c)

Figure 8: (a)-(c) phases of the capillarity test carried out on R-SBgC/PCL composite scaffold.

whilst the precipitation of $\mathrm{Ca}$ and $\mathrm{P}$ were related to $\mathrm{HA}$ formation. By 21 days immersion, a considerable amount of $\mathrm{Ca}$ was precipitated as the dominant phase in comparison with $\mathrm{P}$ according to EDX micrograph and data illustrated in Figure 6(d). The 20\% (w/w) R-SBgC/PCL scaffold demonstrated precipitation of $\mathrm{Ca}, \mathrm{P}, \mathrm{Na}, \mathrm{CL}$, and $\mathrm{Si}$ simultaneously by 14 days of SBF immersion (Figure 6(e)). An increasing amount of $\mathrm{Ca}$ and $\mathrm{P}$ precipitation in the form of HA clusters was revealed after 21 days of SBF immersion as illustrated in Figure 6(f). SEM and EDX analyses after immersion in SBF demonstrated significant changes in the $30 \%(w / w) ~ R-$ $\mathrm{SBgC/PCL}$ scaffold as shown in Figures 6(g) and 6(h).The scaffold surfaces were fully covered by $\mathrm{Ca}$ and $\mathrm{P}$ precipitation by 14 and 21 days indicating HA formation. For 30\% (w/w) R$\mathrm{SBgC/PCL}$ scaffold, the $\mathrm{CaCl}_{2}$ precipitation was significantly reduced and increased peaks of $\mathrm{Ca}$ and $\mathrm{P}$ were revealed after 21 days as illustrated in Figure 6(h). An increase in Ca and $\mathrm{P}$ with reduced $\mathrm{Si}$ indicates the presence of apatite formation according to previous research findings by Liu et al. [28]. SEM micrograph with higher magnification (10K X), as shown in upper left part of Figure 6(h), demonstrated small HA crystals appearing as needles-like structures on the scaffold surface. The $\mathrm{Ca} / \mathrm{P}$ ratio was calculated using the EDX data from different samples and sites and it provided ratio values of $\mathrm{Ca} / \mathrm{P}$ of between 1.57 and 2.6 indicating that the dominant phase of the tested material contains nonstoichiometric $\mathrm{HA}\left(\mathrm{Ca}_{10}\left(\mathrm{PO}_{4}\right)_{6}(\mathrm{OH})_{2}\right)$. Hydroxy apatite was the dominant phase for samples with $1.6 \mathrm{Ca} / \mathrm{P}$ ratio. However, for scaffolds with 2.0 and $2.5 \mathrm{Ca} / \mathrm{P}$ ratios, the $\mathrm{CaO}$ phase started to become visible in the HA phase [29]. The difference can be associated 
with the biphasic calcium phosphate structure formation where $\beta$-TCP and HA phases were present [28]. Ca/P ratio

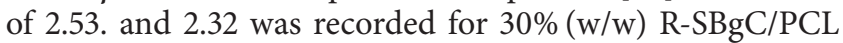
scaffold for 14 and 21 days, respectively. According to previous in vitro study report, the osteoblast cells adhesion on calcium phosphates with higher $\mathrm{Ca} / \mathrm{P}$ ratios up to 2.5 was increased significantly [28].

According to Hong et al., the formation of bone-like apatite on PLLA/(30 wt.\%) bioactive glass ceramic (BGC) composite is at a slower rate than PLLA/(20wt.\%) BGC composite. This may be attributed to the overloading of BGC in the composite [12]. Conversely, according to findings in this study, the $30 \%(\mathrm{w} / \mathrm{w}) \mathrm{R}-\mathrm{SBgC} / \mathrm{PCL}$ scaffold revealed better bioactivity results in comparison to $20 \%(\mathrm{w} / \mathrm{w}) \mathrm{R}$ $\mathrm{SBgC/PCL}$ scaffold which is probably related to homogenous distribution of R-SBgC particles and less agglomerated clusters in the fabricated scaffolds in this study. This finding indicates that the high content of $\mathrm{R}-\mathrm{SBgC}$ addition up to $30 \%$ does not adversely influence the bioactivity of composite scaffold.

\section{Conclusions}

The highly porous composite scaffolds based on R-SBgC/PCL in this study were fabricated using TIPS technique. R-SBgC particles were successfully incorporated into the composite scaffold providing interconnected porous structures of approximately $80 \%$ and an average pore size of $100 \mu \mathrm{m}$. In vitro test showed the formation of $\mathrm{HA}$ on the surface of the scaffold, with the highest formation of HA observed in 30\% (w/w) R-SBgC/PCL composite scaffolds. The porosities, densities, and pore sizes of the produced scaffolds varied with $\mathrm{R}-\mathrm{SBgC}$ concentrations. The interconnected pore structure of the scaffold was indicated by means of capillarity test using inked liquid. R-SBgC nanoparticles incorporated within the scaffolding material improved the compressive mechanical properties. However, in order for this material to be used for bone tissue engineering, further studies investigating the osteoconductive behavior need to be conducted. In addition, other studies which may include material degradation rate, cell growth and adhesion, biocompatibility, and material toxicity would need to be carried out in order to further establish the material being produced as a potential biomaterial. The present study proved that the use of rice husk, which is an agricultural waste, in bioglass enhances PCL properties for applications in bone tissue replacement.

\section{Conflict of Interests}

The authors certify that there is no conflict of interests regarding the publication of this paper.

\section{Acknowledgments}

The authors acknowledge the Universiti Teknologi Malaysia (UTM), the Research Management Center (RMC), the Ministry of Higher Education (MOHE), and the Faculty of Bioscience and Medical Engineering (FBME). This research was supported by UTM research Grants (vote: 79412 and $4 \mathrm{~F} 126,03 \mathrm{H} 13)$. One of the authors of this paper is supported by the University of Malaya HIR-MOHE research grant initiative.

\section{References}

[1] L. G. Griffith and G. Naughton, "Tissue engineering-current challenges and expanding opportunities," Science, vol. 295, no. 5557, pp. 1009-1014, 2002.

[2] R. A. Bhatt and T. D. Rozental, "Bone graft substitutes," Hand Clinics, vol. 28, no. 4, pp. 457-468, 2012.

[3] S. K. Nandi, S. Roy, P. Mukherjee, B. Kundu, D. K. De, and D. Basu, "Orthopaedic applications of bone graft \& graft substitutes: a review," Indian Journal of Medical Research, vol. 132, no. 7, pp. 15-30, 2010.

[4] E. Tamjid, R. Bagheri, M. Vossoughi, and A. Simchi, "Effect of particle size on the in vitro bioactivity, hydrophilicity and mechanical properties of bioactive glass-reinforced polycaprolactone composites," Materials Science and Engineering C, vol. 31, no. 7, pp. 1526-1533, 2011.

[5] M. A. Woodruff and D. W. Hutmacher, "The return of a forgotten polymer-polycaprolactone in the 21st century," Progress in Polymer Science, vol. 35, no. 10, pp. 1217-1256, 2010.

[6] A. R. Boccaccini and J. J. Blaker, "Bioactive composite materials for tissue engineering scaffolds," Expert Review of Medical Devices, vol. 2, no. 3, pp. 303-317, 2005.

[7] A. R. Boccaccini, M. Erol, W. J. Stark, D. Mohn, Z. Hong, and J. F. Mano, "Polymer/bioactive glass nanocomposites for biomedical applications: a review," Composites Science and Technology, vol. 70, no. 13, pp. 1764-1776, 2010.

[8] J.-H. Jo, E.-J. Lee, D.-S. Shin et al., "In vitro/in vivo biocompatibility and mechanical properties of bioactive glass nanofiber and poly( $\varepsilon$-caprolactone) composite materials," Journal of Biomedical Materials Research B, vol. 91, no. 1, pp. 213-220, 2009.

[9] P. X. Ma, R. Zhang, G. Xiao, and R. Franceschi, "Engineering new bone tissue in vitro on highly porous $\operatorname{poly}(\alpha$-hydroxyl acids)/hydroxyapatite composite," Journal of Biomedical Materials Research, vol. 54, pp. 284-293, 2001.

[10] D. Arcos and M. Vallet-Regí, "Sol-gel silica-based biomaterials and bone tissue regeneration," Acta Biomaterialia, vol. 6, no. 8, pp. 2874-2888, 2010.

[11] V. Maquet, A. R. Boccaccini, L. Pravata, I. Notingher, and R. Jérôme, "Porous poly $(\alpha$-hydroxyacid)/bioglass composite scaffolds for bone tissue engineering. I: preparation and in vitro characterisation," Biomaterials, vol. 25, no. 18, pp. 4185-4194, 2004.

[12] Z. Hong, R. L. Reis, and J. F. Mano, "Preparation and in vitro characterization of scaffolds of poly(l-lactic acid) containing bioactive glass ceramic nanoparticles," Acta Biomaterialia, vol. 4, no. 5, pp. 1297-1306, 2008.

[13] A. M. El-Kady, E. A. Saad, B. M. A. El-Hady, and M. M. Farag, "Synthesis of silicate glass/poly(l-lactide) composite scaffolds by freeze-extraction technique: characterization and in vitro bioactivity evaluation," Ceramics International, vol. 36, no. 3, pp. 995-1009, 2010.

[14] B. Lei, K. H. Shin, D. Y. Noh et al., "Sol-gel derived nanoscale bioactive glass (NBG) particles reinforced poly( $\varepsilon$-caprolactone) composites for bone tissue engineering," Materials Science and Engineering C, vol. 33, pp. 1102-1108, 2013. 
[15] B. A. Allo, A. S. Rizkalla, and K. Mequanint, "Synthesis and electrospinning of $\varepsilon$-polycaprolactone-bioactive glass hybrid biomaterials via a sol-gel process," Langmuir, vol. 26, no. 23, pp. 18340-18348, 2010.

[16] Y. S. Nam and T. G. Park, "Biodegradable polymeric microcellular foams by modified thermally induced phase separation method," Biomaterials, vol. 20, no. 19, pp. 1783-1790, 1999.

[17] J. P. Nayak, S. Kumar, and J. Bera, "Sol-gel synthesis of bioglassceramics using rice husk ash as a source for silica and its characterization," Journal of Non-Crystalline Solids, vol. 356, no. 28-30, pp. 1447-1451, 2010.

[18] N. Yalçin and V. Sevinç, "Studies on silica obtained from rice husk," Ceramics International, vol. 27, no. 2, pp. 219-224, 2001.

[19] Y. Y. Hsu, J. D. Gresser, D. J. Trantolo, C. M. Lyons, P. R. J. Gangadharam, and D. L. Wise, "Effect of polymer foam morphology and density on kinetics of in vitro controlled release of isoniazid from compressed foam matrices," Journal of Biomedical Materials Research, vol. 35, no. 1, pp. 107-116, 1997.

[20] T. Kokubo and H. Takadama, "How useful is SBF in predicting in vivo bone bioactivity?" Biomaterials, vol. 27, no. 15, pp. 29072915, 2006.

[21] N. Sultana and T. H. Khan, "In vitro degradation of PHBV scaffolds and nHA/PHBV composite scaffolds containing hydroxyapatite nanoparticles for bone tissue enginering," Journal of Nanomaterials, vol. 1, pp. 1-12, 2012.

[22] S. I. Roohani-Esfahani, S. Nouri-Khorasani, Z. F. Lu, R. C. Appleyard, and H. Zreiqat, "Effects of bioactive glass nanoparticles on the mechanical and biological behavior of composite coated scaffolds," Acta Biomaterialia, vol. 7, no. 3, pp. 1307-1318, 2011.

[23] K. Zhang, Y. Wang, M. A. Hillmyer, and L. F. Francis, "Processing and properties of porous poly(L-lactide)/bioactive glass composites," Biomaterials, vol. 25, no. 13, pp. 2489-2500, 2004.

[24] V. Cannillo, F. Chiellini, P. Fabbri, and A. Sola, "Production of Bioglass 45S5-polycaprolactone composite scaffolds via saltleaching," Composite Structures, vol. 92, no. 8, pp. 1823-1832, 2010.

[25] X. Li, J. Shi, X. Dong, L. Zhang, and H. Zeng, "A mesoporous bioactive glass/polycaprolactone composite scaffold and its bioactivity behavior," Journal of Biomedical Materials Research A, vol. 84, pp. 84-91, 2008.

[26] K. Rezwan, Q. Z. Chen, J. J. Blaker, and A. R. Boccaccini, "Biodegradable and bioactive porous polymer/inorganic composite scaffolds for bone tissue engineering," Biomaterials, vol. 27, no. 18, pp. 3413-3431, 2006.

[27] D. Bellucci, V. Cannillo, G. Ciardelli, P. Gentile, and A. Sola, "Potassium based bioactive glass for bone tissue engineering," Ceramics International, vol. 36, no. 8, pp. 2449-2453, 2010.

[28] H. Liu, H. Yazici, C. Ergun, T. J. Webster, and H. Bermek, "An in vitro evaluation of the $\mathrm{Ca} / \mathrm{P}$ ratio for the cytocompatibility of nano-to-micron particulate calcium phosphates for bone regeneration," Acta Biomaterialia, vol. 4, no. 5, pp. 1472-1479, 2008.

[29] C. Ergun, H. Liu, T. J. Webster, E. Olcay, Ş. Yilmaz, and F. C. Sahin, "Increased osteoblast adhesion on nanoparticulate calcium phosphates with higher $\mathrm{Ca} / \mathrm{P}$ ratios," Journal of Biomedical Materials Research A, vol. 85, no. 1, pp. 236-241, 2008. 

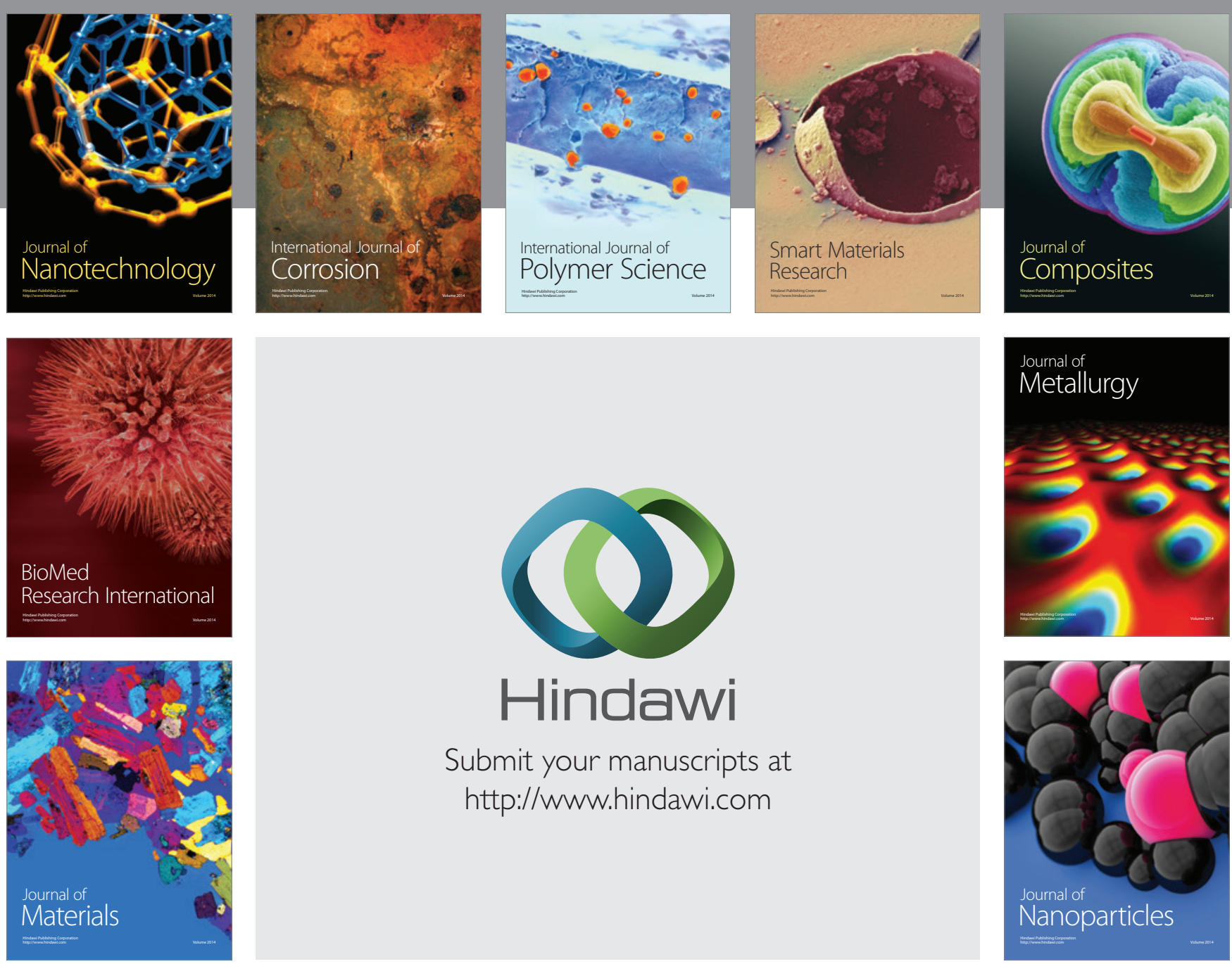

Submit your manuscripts at http://www.hindawi.com
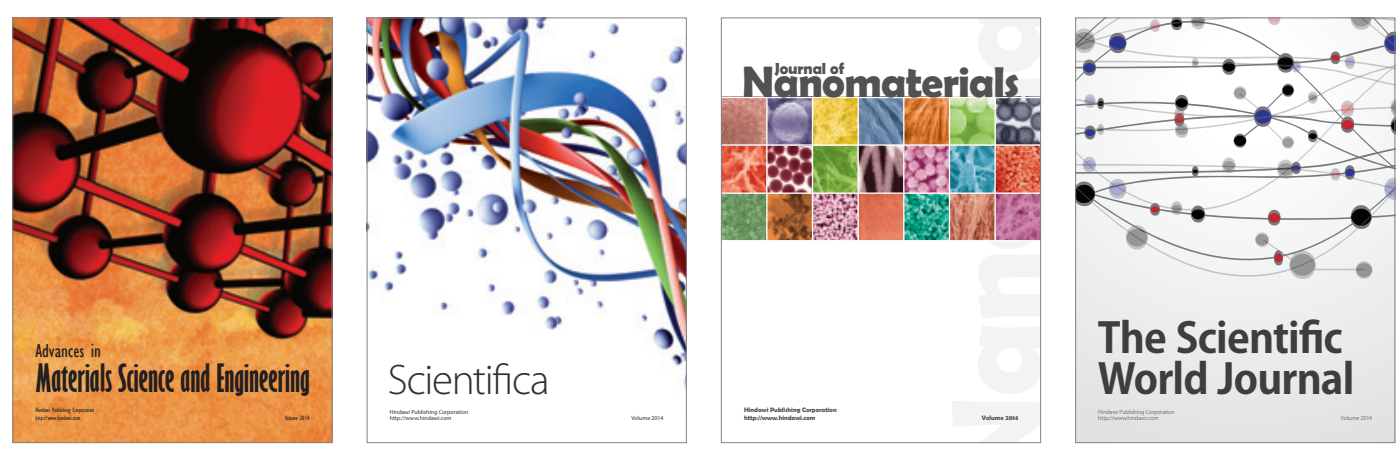

\section{The Scientific World Journal}
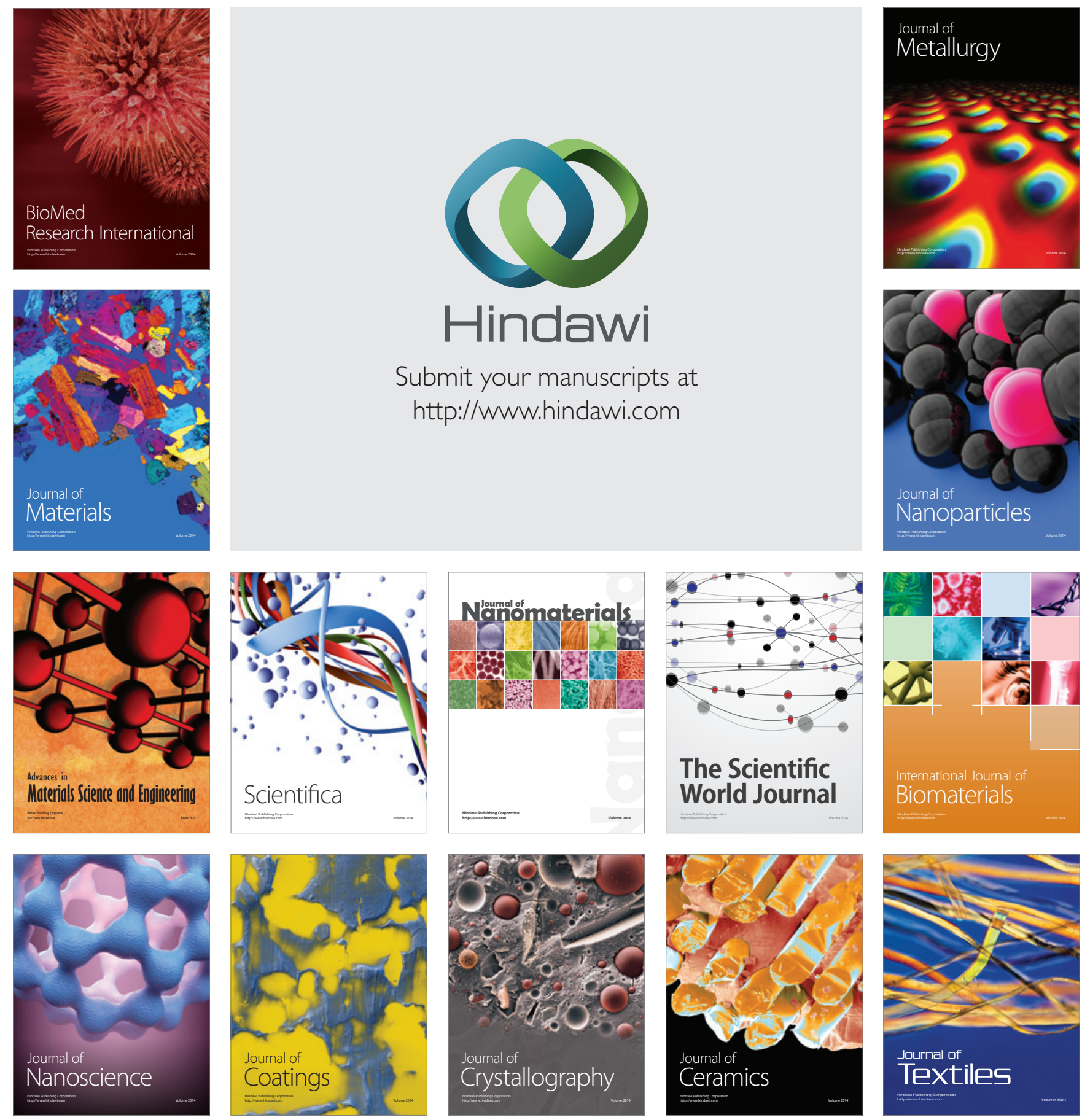